\title{
Group cohesion in group-based personal practice
}

\author{
Ulrike Maaß $^{1 \star}$ (D), Franziska Kühne ${ }^{1}$ (D), Daniela Hahn² ${ }^{2}$ and Florian Weck ${ }^{1}$ \\ ${ }^{1}$ University of Potsdam, Potsdam, Germany and ${ }^{2}$ Johannes Gutenberg University Mainz, Mainz, Germany \\ *Corresponding author. Email: ulrikemaass@uni-potsdam.de
}

(Received 17 May 2021; revised 20 July 2021; accepted 27 July 2021; first published online 27 September 2021)

\begin{abstract}
Background Personal practice (PP) is an integral component of many psychotherapy training programmes. It aims to promote personal and professional growth and is often conducted in a group format (g-PP). Group cohesion is one of the most researched mechanisms in group psychotherapy, but has rarely been studied in the context of g-PP.

Aims and method This exploratory study examines the associations between cohesion, satisfaction with g-PP, its impact on personal and professional development, and theoretical orientation in a sample of $n=329$ German psychotherapy trainees. Cohesion was assessed with the group questionnaire (GQ-D; Positive Bonding, Positive Working, Negative Relationship).

Results Overall, participants reported high levels of all outcome variables. Positive Bonding was the strongest predictor of satisfaction with g-PP $(\beta=0.46, p<.001)$. While trainees in cognitive behaviour therapy reported significantly better cohesion scores $(d \geq 0.31)$, trainees in psychodynamic therapy reported significantly higher satisfaction with g-PP and its impact on their developments $(d \geq 0.30)$.

Conclusions Group cohesion appears to be an important factor in g-PP that should be actively promoted by group leaders. However, longitudinal study designs are needed to better understand the emergence of cohesion in $\mathrm{g}-\mathrm{PP}$ as well as potential moderating factors.
\end{abstract}

Keywords: personal therapy; self-reflection; self-practice; therapist effects; therapist training; supervision

\section{Introduction}

Over the past 100 years, several researchers and practitioners have recommended including selfreflection or personal practice (PP) in the training of psychotherapists in order to foster skills development and personal growth (Bennett-Levy, 2019; Freud, 1910; Kanfer et al., 2012; Orlinsky et al., 2001). Freud (1910) even doubted the competence of psychoanalysts who failed to 'achieve anything in such a self-analysis' (p. 1). In fact, the positive impact of PP on therapists is well established (Geller et al., 2005). For example, PP was ranked as the third most important factor in therapist development in a survey among 4000 psychotherapists (Orlinsky et al., 2001). By contrast, the evidence supporting the relationship between PP and patient outcomes is still mixed (Orlinsky et al., 2005). It is worth mentioning that several terms are associated with PP in the literature, such as self-therapy, which focuses on dealing with personal difficulties and aims to promote personal growth, self-practice/self-reflection programs (SP/SR), which focus on practising certain therapy techniques on oneself, meditation-based programmes, which focus on practising mindfulness, and experiential groups, which focus on increasing awareness of one's impact on others and the ability to provide and receive feedback (Bennett-Levy, 2019; Bennett-Levy and Finlay-Jones, 2018; Kivlighan et al., 2019; Malikiosi-Loizos, 2013). The present article aims to shed light on the

(C) The Author(s), 2021. Published by Cambridge University Press on behalf of the British Association for Behavioural and Cognitive Psychotherapies. This is an Open Access article, distributed under the terms of the Creative Commons Attribution licence (https:// creativecommons.org/licenses/by/4.0/), which permits unrestricted re-use, distribution, and reproduction in any medium, provided the original work is properly cited. 
group-based form of PP (g-PP) and the use of group cohesion as one of its potentially effective mechanisms. Because this is an exploratory study on this subject, we do not limit g-PP therapy to any particular approach (e.g. personal growth, self-practice, mindfulness).

\section{Theoretical model of personal practice}

Bennett-Levy (2019) defines PP as 'formal psychological interventions and techniques that therapists engage with self-experientially over an extended period of time (weeks, months or years) as individuals or groups, with a reflective focus on their personal and/or professional development' (p. 133). Bennett-Levy and Finlay-Jones (2018) have developed a theoretical framework for PP based on cognitive behavioral therapy (CBT). According to the model, there are four types of motivations to engage in PP: personal problems, personal growth, selfcare, and therapist skill development. Furthermore, PP usually intends to foster personal development and well-being, self-awareness, interpersonal beliefs, attitudes and skills, reflective skills, and conceptual/technical skills. In addition, PP is characterized by reflection about the personal and/or therapist self as its key process. The main assumption of the model is that the connection between personal and therapeutic self-reflection is necessary for PP to be effective. This process is referred to as crossing 'the reflective bridge' and describes the reflection on the implications of PP for one's therapist self. One prediction of the model is that self-therapy affects the personal-self more than the therapist-self. In contrast, SP/SR programmes, for example, affect both the personal and therapist self, but focus more on the therapist-self, because they support the therapists in dealing with personal difficulties only insofar as it also helps to improve professional behaviour and promotes a deeper understanding of client processes (Chigwedere et al., 2020).

\section{Group-based personal practice}

Participation in a self-reflective group is an integral part of the education of group psychotherapists (Bennett-Levy and Finlay-Jones, 2018), and is recommended by the corresponding professional associations, such as the Association for Specialists in Group Work (McCarthy et al., 2014). Like individual PP, there are several labels for group-based PP, ranging from self-reflection group, experiential or growth group, experiential group psychotherapy, to small group activity (Zhu, 2018).

Generally, surveys and interview studies have revealed that group participants associate positive expectations with g-PP before (Moller and Rance, 2013) and after the actual group experience, with 77-97\% of trainees evaluating g-PP as useful (Anderson and Price, 2001). Usually, trainees indicate that $\mathrm{g}$-PP has positive effects on their self-awareness, communication style, interpersonal behaviour, self-acceptance, acceptance of others, empathy, theory of mind, personal sensitivity, insights regarding strengths and areas for growth, and a deeper theory-practice connection (Ieva et al., 2009; Kline et al., 1997; Schneider and Rees, 2012). One study compared g-PP with individual PP in a quasi-experimental design consisting of two samples of counselling trainees (Chigwedere et al., 2020). An individual PP condition $(n=34)$ was compared with a g-PP condition $(n=41)$ created as a self-practice/self-reflection programme, including 'group-based reflections' in addition to the self-practice of CBT interventions. The results demonstrated that structured g-PP was perceived as influencing personal and therapist development as well as empathy, significantly more than individual PP, with medium to large effect sizes (Chigwedere et al., 2020).

Despite these promising results on $\mathrm{g}$-PP, the studies cited above are mainly based on small sample sizes ( $n=11$ to 25; Moller and Rance, 2013; Schneider and Rees, 2012), qualitative methods (e.g. interviews) or self-report surveys, and thus, the research on g-PP is still considered sparse and by no means exhaustive (Young et al., 2013). Furthermore, there is 
only limited knowledge on the effective mechanisms of g-PP, that is, the 'processes, dynamics, member development, and group development that occur within such groups' (Ohrt et al., 2014; p. 215). However, understanding the effective factors in g-PP is important, in order to be able to decide when and for whom g-PP or individual PP could be most useful.

\section{Effective factors in group-based personal practice}

Bennett-Levy and Finlay-Jones (2018) suggest that different individuals might benefit differently from individual and group experiences. Several authors emphasize the importance of groupspecific factors that enhance learning above and beyond the possibilities of an individual setting. For example, the use of peer feedback in g-PP is considered helpful for identifying dysfunctional therapist assumptions that might impede the work with clients (Yalom and Leszcz, 2008). Other group-specific factors in g-PP concern transference learning, opportunities for interpersonal behaviour change, observations of group processes, understanding how impressions are formed, learning through modelling, and using the group as a support system (Ieva et al., 2009; Kline et al., 1997; Schneider and Rees, 2012; Tschuschke et al., 2011; Young et al., 2013).

One of the most frequently studied mechanisms in group psychotherapy is cohesion, which refers to the therapeutic relationship in group psychotherapy (Burlingame et al., 2001). This includes a member's sense of belonging and individual commitment to the group, but also mutual trust, support and a collective commitment to learning within the group (Burlingame et al., 2001). While vertical cohesion is described as containing the member-leader relationship (i.e. perceived leaders' competence, warmth, etc.), horizontal cohesion refers to the member-member and member-group relationship (e.g. interpersonal and emotional support; Burlingame et al., 2001). Thus, cohesion encompasses several relationships that require paying attention not only to the growth of individual group members, but also to the group as a whole (Burlingame et al., 2001). Two meta-analyses have confirmed the positive impact of cohesion on therapy outcomes, with average correlations of $r=0.25-0.26$, based on up to 55 studies and 6.055 clients (Burlingame et al., 2011; Burlingame et al., 2018). Moreover, the strength of the cohesion-outcome relationship varies between moderator variables, such as the type of group and theoretical orientation, being strongest for relatively unstructured groups $(r=0.36-0.38)$ and interpersonal groups $(r=0.48-0.58)$. Psychodynamic and CBT groups achieved positive but lower coefficients $(r=0.18-0.27)$.

Cohesion is not only one of the most effective aspects in group therapy, but may also be relevant for g-PP. More specifically, numerous qualitative studies have revealed that cohesion, just as in group psychotherapy, elicits disclosure and connection among trainees, develops their empathy, and increases over time in g-PP (Kivlighan et al., 2019; Luke and Kiweewa, 2010; Oh et al., 2018; Sunderji et al., 2013; Young et al., 2013). For example, Kivlighan et al. (2019) interviewed $n=8$ psychology students, enrolled in a group leadership course, after their participation in a 13-week experiential training group. The authors found that both higher in-session and higher cross-session cohesion were related to perceived social learning and interpersonal awareness.

In sum, g-PP is positively evaluated by trainees, and group cohesion seems to be an important impact factor for personal or professional growth. Nonetheless, most of the literature is based on group psychotherapy training promoting experiential learning for subsequent work as a group leader (McCarthy et al., 2014). However, g-PP might be useful not only for future group leaders, but also in the general training of therapists (Tschuschke et al., 2011). In many training programmes, the group is the preferred setting for PP (Laireiter and Willutzki, 2003), which may be more cost- and time-effective than individual PP. However, little attention has yet been paid to the usefulness and limitations of g-PP as a training method, and to its effective mechanisms, such as cohesion. Furthermore, it is unclear whether there are 
Table 1. Sample statistics

\begin{tabular}{lc}
\hline & $n(\%)$ \\
\hline Female & $292(88.8)$ \\
Age (mean, SD) & $31.73(6.40)$ \\
Theoretical orientation & $219(66.6)$ \\
CBT & $97(29.5)$ \\
PDT & $13(4.0)$ \\
Other & \\
PP is mandatory in curriculum & $288(87.5)$ \\
g-PP & $146(44.4)$ \\
i-PP & \\
Most frequent topics in PP* & $284(87.1)$ \\
Biography & $271(83.1)$ \\
Own topics & $242(74.2)$ \\
Own processes (e.g. interaction, cognition, emotion) & \\
Most frequent techniques in PP* & $224(68.7)$ \\
Feedback & $220(67.5)$ \\
Personal self-reflection & $211(64.7)$ \\
Practise therapeutic methods on oneself & Mean (SD) \\
Experience in hours & $1771.05(632.44)$ \\
Clinical & $85.65(42.74)$ \\
g-PP & $39.18(93.24)$ \\
i-PP &
\end{tabular}

$N$ values range from 320 to 329 due to occasional missing data. CBT, cognitive behavioural therapy; PDT, psychodynamic therapy; g-PP, group-based personal practice; i-PP, individual personal practice. *Participants who indicated that the according topic or technique was addressed 'sometimes' or 'often'.

moderators for the cohesion-outcome relationship in $\mathrm{g}$-PP, for example, the theoretical orientation.

\section{Objectives}

The present study aimed to examine group cohesion in g-PP among a sample of German psychotherapists in training (i.e. general training, no group leader training), and with various theoretical backgrounds. The research questions were: (1) how satisfied are trainees with their current g-PP?; (2) how do they perceive the level of group cohesion?; (3) is there a correlation between cohesion and the satisfaction with g-PP?; and (4) are there any differences regarding perceived group cohesion between theoretical orientations?

\section{Method}

\section{Recruitment, eligibility criteria and sample}

Between January and February 2020, 468 psychotherapists in training were recruited by contacting 210 training institutes across Germany via email for a larger online study of which the current study is part. The protocol for the larger study was approved by the ethics committee of the Department of Psychology at the Johannes Gutenberg University Mainz (application 2018-JGU-psychEK-028-X1). In addition, the protocol for the current study was registered with the Open Science Framework (osf.io/3ukbs) on 18 March 2021. The inclusion criteria were (1) participation in psychotherapy training ( $n=1$ was excluded), (2) at least one previous individual or g-PP session ( $n=56$ were excluded), and (3) informed consent $(n=1$ was excluded). For the purposes of this study, we analysed a subset of the data, including only those participants with experience in g-PP. The characteristics of the final sample $(N=329)$ are displayed in Table 1 . Almost $88 \%$ of trainees indicated that g-PP was mandatory in their 
curriculum, as opposed to individual PP, which was obligatory for $44 \%$ of the sample. On average, participants had already attended at least 85.65 hours of g-PP $(S D=42.74)$.

\section{Group-based personal practice}

The training for psychotherapists in Germany is legally regulated and consists of theoretical education ( $\geq 600$ hours), clinical work ( $\geq 1800$ hours), treatment of patients under supervision ( $\geq 600$ hours), and at least 120 hours of PP (PsychTh-APrV, 1998). According to this regulation, the goal of $\mathrm{PP}$ is 'the reflection or modification of personal prerequisites for therapeutic experience and action, including biographical aspects, as well as essential aspects of experience and action related to the therapeutic alliance and personal development in the course of training' ( $\$ 5$; translated by first author). This description is in line with the general definition of PP stated above including self-reflection as the key process. Furthermore, personal growth can be named as the strongest motivation and personal development as the intended outcome of PP (cf. Bennett-Levy and Finlay-Jones, 2018).

However, the regulation does not describe how PP should be implemented in terms of specific methods (e.g. self-therapy, SP/SR, meditation, experiential group). Table 1 lists the three most common topics and methods used in this sample (for more details on the characteristics of PP in the overall sample, see Hahn et al., in prep.). Between 74.2 and $87.1 \%$ of the participants reported that g-PP included biography work, individual topics, and the recognition of individual patterns (sometimes or often). Furthermore, between 64.7 and $68.7 \%$ indicated that feedback, personal reflection, and self-practice of therapeutic methods were the most common techniques. More information on the differences between CBT and psychodynamic orientations are displayed in the Supplementary material online and in Hahn et al. (in prep.). For example, the topic 'goals and agreements' was more often used in CBTthan among psychodynamic therapy (PDT)-oriented g-PP $\left(53.2\right.$ vs $37.1 \% ; \chi^{2}(1,313)=6.34$, $p=0.012)$. In contrast, topics involving interaction with group members were more common for PDT than for CBT trainees, for example 'self- $v s$ other perception' (67.0 vs 47.7\%, $\left.\chi^{2}(1,313)=9.29, p=0.002\right)$ and 'group cohesion' (53.6 vs 36.6\%, $\chi^{2}(1,313)=7.29, p=0.007$; see Supplementary material).

Given this information, we assume that the majority of g-PP is currently a mixture of selftherapy, SP/SR programmes, and experiential groups with a stronger focus on the personal self than on the therapist self.

\section{Measurements}

Personal practice

Satisfaction with g-PP and its impact on personal and professional development was assessed via two items: (1) 'Please indicate how satisfied you have been so far with your g-PP in the training in terms of developing your personal and therapeutic skills'; rating scale from 1 ('not at all') to 5 ('very'); (2) 'What impact did your g-PP have on (a) your overall personal development, and (b) your overall therapeutic skills?'; rating scale from 1 ('negative') to 5 ('positive').

\section{Group cohesion}

The German version of the Group Questionnaire (GQ-D; Bormann et al., 2011; Krogel, 2009) for the assessment of cohesion consists of the subscales Positive Bonding, Positive Working, and Negative Relationship, which can be further divided into member-leader, member-member, and member-whole-group subscales. In order to give the study a clear focus, we used the member-whole-group subscale only. The Cronbach's alpha values for the current sample were: 0.91 for Positive Bonding (five items, e.g. 'The members liked and cared about each 
Table 2. Descriptive statistics for group cohesion, satisfaction with g-PP, and the impact on personal or professional development

\begin{tabular}{lc}
\hline & Mean (SD) \\
\hline Group cohesion & \\
Positive Bonding & $5.93(1.20)$ \\
Positive Working & $4.82(1.33)$ \\
Negative Relationship & $2.56(1.25)$ \\
Impact on development & \\
Personal & $4.07(0.79)$ \\
Professional & $4.03(0.78)$ \\
Overall satisfaction & $3.76(1.14)$ \\
Positive impact on development & $\boldsymbol{n}(\%)$ \\
Personal & $255(78.7)$ \\
Professional & $241(74.4)$ \\
Overall satisfaction & $198(61.9)$ \\
\hline
\end{tabular}

$N$ values range from 320 to 325 due to occasional missing data.

other'), 0.83 for Positive Working (four items, e.g. 'The other group members and I are working together toward mutually agreed upon goals'), and 0.71 for Negative Relationship (three items; e.g. 'There was tension and anxiety between the members').

\section{Analytical strategy}

The analyses were done using RStudio 1.1.456 (RStudio Team, 2015). We calculated descriptive statistics and bivariate correlations for the outcome variables (i.e. cohesion, satisfaction, personal and professional development). A multiple regression was calculated using the GQ-D subscales as predictors for the overall satisfaction with the g-PP, controlling for the number of already completed g-PP hours. Due to the unbalanced sample sizes across the theoretical orientations, we pooled participants from psychoanalytical and/or psychodynamic orientations into one group (PDT; $n=97)$, and excluded participants with a systemic $(n=6)$ or other background $(n=7)$. Thus, group comparisons (i.e. CBT vs PDT) were conducted using a series of twotailed $t$-tests, applying a Bonferroni correction to the significance level of .05. Finally, a moderator analysis examined whether the relationship between cohesion (i.e. GQ-D sum score) and satisfaction with g-PP differed between theoretical orientations (CBT vs PDT), controlling for hours of already completed g-PP.

\section{Changes to the pre-registration}

The description of the topics and techniques in g-PP, the $\chi^{2}$ tests, moderation analysis, and twotailed $t$-tests are deviations from the pre-registration. These changes were made in response to the data structure or during the revision process.

\section{Results}

\section{How satisfied are trainees with their current group-based personal practice?}

Overall, trainees reported being very satisfied with their g-PP (Table 2) and between 74.4 and $78.7 \%$ of the sample noted a rather positive impact on their personal and professional development (i.e. chose the 'positive' or 'rather positive' category). Only a minority indicated negative impacts on their personal $(n=10,3.1 \%)$ or professional development $(n=5,1.5 \%)$. 
Table 3. Correlations between the study variables

\begin{tabular}{|c|c|c|c|c|c|}
\hline & Bonding & $\begin{array}{l}\text { Negative } \\
\text { relationship }\end{array}$ & $\begin{array}{c}\text { Personal } \\
\text { development }\end{array}$ & $\begin{array}{l}\text { Professional } \\
\text { development }\end{array}$ & Satisfaction \\
\hline Positive Working & $0.56^{\star \star \star}$ & $-0.44^{\star \star \star}$ & $0.34^{\star \star \star}$ & $0.29^{\star \star \star}$ & $0.37^{\star \star \star}$ \\
\hline Positive Bonding & & $-0.68^{\star \star \star}$ & $0.44^{\star \star \star}$ & $0.35^{\star \star \star}$ & $0.51^{\star \star \star}$ \\
\hline Negative Relationship & & & $-0.24^{\star \star \star}$ & $-0.18^{\star \star}$ & $-0.34^{\star \star \star}$ \\
\hline Personal development & & & & $0.67^{\star \star \star}$ & $0.69^{\star \star \star}$ \\
\hline Professional development & & & & & $0.63^{\star \star \star}$ \\
\hline
\end{tabular}

$N$ values range from 312 to 329 due to single missing data. ${ }^{* *} p<.01 .{ }^{* \star *} p<.001$.

\section{How do they perceive the level of group cohesion?}

Trainees perceived strong cohesion in terms of Positive Bonding and Working and low levels of Negative Relationship (Table 2).

\section{Is there a correlation between cohesion and the satisfaction with group-based personal practice?}

As Table 3 indicates, all cohesion subscales were significantly associated with a positive impact on personal or professional development, whereby Positive Bonding achieved the highest correlations $(r \geq .35, p<.001)$. Similarly, the multiple regression revealed that Positive Bonding was the strongest predictor for the overall satisfaction with g-PP, controlling for the number of already completed g-PP hours: $\quad \beta_{\text {bonding }}=0.46, \quad p<.001 ; \quad \beta_{\text {working }}=0.14, \quad p=.015$; $\beta_{\text {neg.relationship }}=3.62, p=.583$, and $\beta_{\text {hours }}=0.09, p=.057$. The model explained $28 \%$ of the variance in overall satisfaction, $F_{4,314}=30.47, p<.001$.

\section{Are there any differences regarding perceived group cohesion between theoretical orientations?}

Figure 1 presents the means for cohesion and satisfaction, with g-PP dependent on trainees' theoretical orientation (i.e. CBT vs PDT). Although g-PP was perceived as highly cohesive in both schools, CBT trainees indicated significantly higher scores for Positive Bonding $\left(t_{157.7}=2.449, p=.009\right)$, Positive Working $\left(t_{166.3}=4.238, p<0.001\right)$ and significantly lower scores for Negative Relationship $\left(t_{181}=-6.183, p<.001\right)$ than PDT trainees, with small to large effect sizes $(d \geq 0.31)$. However, trainees in PDT were significantly more satisfied with g-PP $\left(t_{165.8}=-2.348, p=.015\right)$ and assigned a significantly stronger impact on their professional development $\left(t_{176.7}=-3.606, p<.001\right)$. The effects were small to moderate $(d \geq 0.30)$. There was no significant difference for personal development $\left(t_{161.9}=-1.849\right.$, $p=.054)$. The moderator analysis revealed a non-significant interaction between the theoretical orientation and the cohesion-satisfaction relationship $\left(\beta_{\mathrm{GQ}-\mathrm{D} \times \mathrm{PDT}}=0.12, p=.056\right)$.

\section{Discussion}

The present article focused on cohesion as a potentially effective mechanism in g-PP. The results indicate that 74.4 to $78.7 \%$ of participants experienced positive impacts of g-PP on their personal or professional development. Furthermore, group cohesion was described as generally high. The subscales Positive Bonding and Positive Working in particular, predicted the overall satisfaction with g-PP, and the whole model explained about $30 \%$ of the variance in the outcome. Finally, while CBT trainees indicated stronger cohesion scores than PDT trainees, PDT trainees reported slightly higher satisfaction with g-PP. However, the theoretical orientation did not significantly moderate the cohesion-satisfaction relationship. 


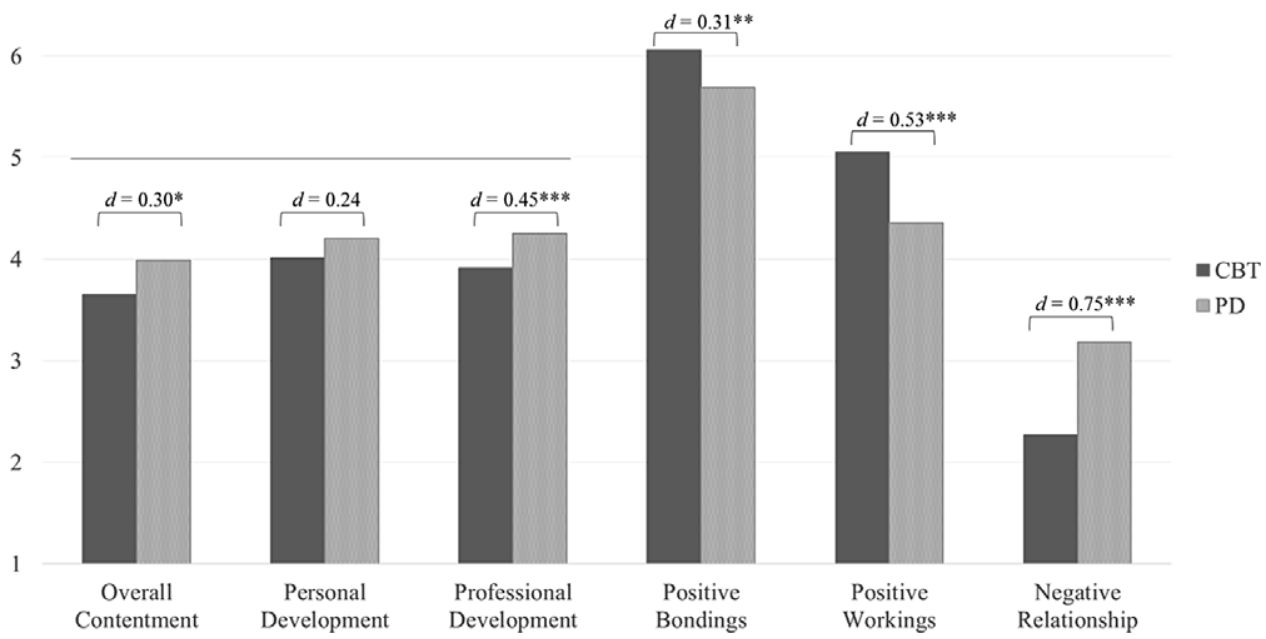

Figure 1. Group comparisons between cognitive behavioural therapy (CBT) and psychodynamic therapy (PD). Left-hand three bars denote experience with group-based personal practice; right-hand three bars denote cohesion subscales. $d$, Cohen's $d$ effect size. ${ }^{\star} p<.05,{ }^{* *} p<.01,{ }^{\star * *} p<.001$.

\section{Cohesion as an effective factor in group-based personal practice}

Our results are in line with a qualitative study showing that high cohesion in g-PP had a positive impact on the group members' engagement in the process (Bennett-Levy and Lee, 2014). Thus, cohesion should be stressed actively by group leaders, as recommended for group therapy (Burlingame et al., 2001). For example, high cohesion is supposed to promote the acceptance of feedback from peers, which, in turn, is associated with increased insight into and motivation for change. Thus, group leaders could foster PP group members' mutual support and ensure that the 'format becomes a curative influence in its own right and not merely a "watered-down" version of individual therapy [...]' (Burlingame et al., 2001; p. 373). Several authors describe cohesion-promoting strategies (Burlingame et al., 2011; Hippler and Görlitz, 2001; Leitemo et al., 2020), which could be adapted to PP and applied whenever difficult group conditions appear. Such interventions include the clarification of expectations and roles, establishment of group structures, encouragement of member interaction, positive feedback, fostering self-disclosure, constructive conflict resolution, and a focus on emotional expression and exchange. Experiential exercises as well as non-judging, open-minded and securely attached behaviours of the group leader can also enhance cohesion (Burlingame et al., 2011; Hippler and Görlitz, 2001; Leitemo et al., 2020). Despite such strategies, there may still be a decline in cohesion that should be addressed. Within the psychotherapy literature, ruptures in the therapeutic alliance are well known and defined as 'a tension or breakdown in the collaborative relationship between patient and therapist' (Safran et al., 2011; p. 80). The positive resolution (i.e. 'repair') of ruptures is associated with better alliance scores and better symptom outcomes in individual therapy (Eubanks et al., 2018). As there are efforts to apply the rupture-repair model to group therapy (Lo Coco et al., 2019), researchers may also consider this aspect in future studies on g-PP and cohesion.

\section{Differences between theoretical orientations}

The group differences between CBT and PDT require further discussion, although these analyses were exploratory and should be interpreted with caution. For example, the fact that CBT trainees 
perceived their g-PP to be more goal-oriented, as indicated by higher Positive Working scores and a more frequent use of 'PP goals and agreements', is in line with the general CBT conceptualization (Kanfer et al., 2012). By contrast, Strauß and Mattke (2018) suspect that conflict plays a greater role in psychodynamic PP groups due to studies reporting a positive association between conflict and therapy outcomes. For example, Tschuschke and Greene (2002) found that g-PP, which was relatively unstructured and focused on the processes in the here and now, was associated with more conflicts at the beginning of the group interaction than a g-PP condition, which was more structured and educative. The authors argued that early conflicts and high engagement can lead to conflict resolution and contribute to a stronger sense of learning about the self and the therapist role. In line with these findings, trainees with a psychodynamic background reported higher conflict levels in this sample, as indicated by higher Negative Relationship scores. Unfortunately, we do not have enough information on the exact methods and processes within each group. However, as topics involving the interaction with group members or others ('self- $v s$ other perception' and 'group cohesion') were also more common among PDT trainees, we speculate that psychodynamic g-PP addressed potential conflicts more explicitly. However, other studies report that more conflicts impede productive work, also in interpretive or supportive PP groups (Ogrodniczuk and Piper, 2003). Thus, the question of how the theoretical orientation influences individual outcomes of g-PP, and of cohesion in particular, warrants further investigation.

\section{Adverse effects}

Despite the positive results, it is also important to understand more about what contributes to the negative effects that some participants noted. Overall, the percentage of those participants was small (i.e. 1.5-3.1\%) and is comparable to the numbers found in previous g-PP studies, ranging between 1 and 10\% (Orlinsky et al., 2005). Potential negative effects usually consist of discomfort, anxiety, power issues, and emotional intensity regarding information sharing in a gPP (Anderson and Price, 2001; Sandell et al., 2006; Schneider and Rees, 2012).

Some authors highlight that mandatory g-PP in particular can have detrimental effects on trainees (e.g. confusion, destabilization), particularly when there is a lack of clear group rationale (Edwards, 2018; Murphy et al., 2018). The majority of participants of the current sample attended mandatory g-PP, but given the low rates of negative effects, and evidence that some mandatory PP programmes can also have positive effects on trainees (Chigwedere et al., 2020), we assume that the obligation itself is a lesser problem.

\section{Limitations and future directions}

The present study aims to draw attention to cohesion as an important factor in g-PP. Nonetheless, there are notable factors that limit its interpretation. Above all, the types of g-PP were not standardized, that is, there was limited information about the concrete methods and procedures within each g-PP at every training institute. Consequently, the results do not allow us to say which g-PP conceptualizations (e.g. self-therapy, SP/SR, meditation) are most strongly correlated with participants' satisfaction or a more positive sense of cohesion. Future studies should compare more standardized programmes against each other. For example, Chigwedere et al. (2020) compared self-therapy with a SP/SR programme and found that participants reported more positive effects on both their therapist self (i.e. interpersonal beliefs, attributes, skills and conceptual/technical skills) but also their personal self (i.e. personal development, wellbeing, and self-awareness) within the SP/SR programme. Similar approaches can be chosen for g-PP. In order to better understand the differences between CBT- and PDT-oriented g-PP, researchers should explicitly outline the focus of these groups (e.g. goal-orientation $v s$ process-orientation). Second, the online survey was brief, 
which is why further studies should gather more information on the specifics of satisfaction with $\mathrm{g}-\mathrm{PP}$, on the factors contributing to the positive and negative impacts of g-PP, and on the member-leader dimension of cohesion. Burlingame et al. (2011, 2018) identified several moderators influencing the cohesion-outcome relationship in group psychotherapies. Future studies on g-PP and cohesion could consider these moderators as well, for example, the group size, the type of group, emphasis on group member interactions, or the number of group sessions. Moreover, other researchers might wish to use validated instruments, for example from the Clinical Outcome Results Standardized Measures (CORE-R) Battery (Burlingame et al., 2006), to assess overall experiences, difficulties, or interpersonal behaviours arising in PP groups. Third, participants in this sample did not provide their answers immediately after their last g-PP, which increases the risk of memory effects. Fourth, as we did not collect information on the cultural backgrounds of the participants, the generalizability of the sample is limited. Finally, as this is a cross-sectional survey, the results are exploratory and warrant further investigation with longitudinal designs.

\section{Conclusion}

The present study asked a large sample of therapist trainees about their experiences with groupbased personal practice and their perceptions of group cohesion. Overall, g-PP was very positively evaluated and group cohesion was high. Furthermore, cohesion was positively associated with the experience of personal and professional development through personal practice. However, more research is needed to better understand the development of cohesion, as well as the variables that influence cohesion over the course of group-based personal practice.

Supplementary material. To view supplementary material for this article, please visit: https://oi.org/10.1017/ S1352465821000369

Acknowledgements. We thank Brian Bloch for professional proofreading.

Author contributions. All authors designed the study. U.M. conducted the statistical analyses, and interpreted the results and wrote the initial draft. All authors critically revised the manuscript, read and approved the final manuscript.

Funding. This research received no specific grant from any funding agency, commercial or not-for-profit sectors.

Conflicts of interest. None.

Ethics statement. The authors have abided by the Ethical Principles of Psychologists and Code of Conduct as set out by the BABCP and BPS The study was approved by the ethics committee of the Johannes Gutenberg University Mainz (application 2018-JGU-psychEK-028-X1). Participants provided written informed consent.

Data availability statement. The data that support the findings of this study are available from the corresponding author, U.M., upon reasonable request. The study protocol and analysis script are openly available in Open Science Framework: Maass, U. (21 March 2021). Self-Practice and Self-Reflection. Retrieved from: osf.io/3ukbs

\section{References}

Anderson, R. D., \& Price, G. E. (2001). Experiential groups in counselor education: student attitudes and instructor participation. Counselor Education and Supervision, 41, 111-119. https://doi.org/10.1002/j.1556-6978.2001.tb01275.x

Bennett-Levy, J. (2019). Why therapists should walk the talk: the theoretical and empirical case for personal practice in therapist training and professional development. Journal of Behavior Therapy and Experimental Psychiatry, 62, 133145. https://doi.org/10.1016/j.jbtep.2018.08.004

Bennett-Levy, J., \& Finlay-Jones, A. (2018). The role of personal practice in therapist skill development: a model to guide therapists, educators, supervisors and researchers. Cognitive Behaviour Therapy, 47, 185-205. https://doi.org/10.1080/ 16506073.2018.1434678

Bennett-Levy, J., \& Lee, N. K. (2014). Self-practice and self-reflection in cognitive behaviour therapy training: what factors influence trainees' engagement and experience of benefit? Behavioural and Cognitive Psychotherapy, 42, 48-64. https://doi. $\operatorname{org} / 10.1017 /$ S1352465812000781 
Bormann, B., Burlingame, G. M., \& Strauß, B. (2011). Der Gruppenfragebogen (GQ-D): Instrument zur Messung von therapeutischen Beziehungen in der Gruppenpsychotherapie. Psychotherapeut, 56, 297-309. https://doi.org/10.1007/ s00278-011-0841-4

PsychTh-APrV (Pub. L. No. Ausbildungs-und Prüfungsverordnung für Psychologische Psychotherapeuten) (1998). https:// www.brd.nrw.de/gesundheit_soziales/LPA-Psychotherapie/pdf-Psychotherapie/PsychTh-APrV.pdf

Burlingame, G. M., Fuhriman, A., \& Johnson, J. E. (2001). Cohesion in group psychotherapy. Psychotherapy: Theory, Research, Practice, Training, 38, 373-379. https://doi.org/10.1037/0033-3204.38.4.373

Burlingame, G. M., McClendon, D. T., \& Alonso, J. (2011). Cohesion in group therapy. Psychotherapy, 48, 34-42. https:// doi.org/10.1037/a0022063

Burlingame, G. M., McClendon, D. T., \& Yang, C. (2018). Cohesion in group therapy: a meta-analysis. Psychotherapy, 55, 384-398. https://doi.org/10.1037/pst0000173

Burlingame, G. M., Strauss, B., Joyce, A., MacNair-Semands, R., MacKenzie, K. R., Ogrodniczuk, J., \& Taylor, S. (2006). CORE Battery-Revised: an assessment tool kit for promoting optimal group selection, process, and outcome. New York, NY: American Group Psychotherapy Association.

Chigwedere, C., Bennett-Levy, J., Fitzmaurice, B., \& Donohoe, G. (2020). Personal practice in counselling and CBT trainees: the self-perceived impact of personal therapy and self-practice/self-reflection on personal and professional development. Cognitive Behaviour Therapy, 1-17. https://doi.org/10.1080/16506073.2020.1846608

Edwards, J. (2018). Counseling and psychology student experiences of personal therapy: a critical interpretive synthesis. Frontiers in Psychology, 9, 1732. https://doi.org/10.3389/fpsyg.2018.01732

Eubanks, C. F., Muran, J. C., \& Safran, J. D. (2018). Alliance rupture repair: a meta-analysis. Psychotherapy, 55, 508-519. http://doi.org/10.1037/pst0000185

Freud, S. (1910). Die zukünftigen Chancen der psychoanalytischen Therapie. Psyalpha. https://www.psyalpha.net/de/themen/ behandlungstechnik/freud-technische-schriften/sigmund-freud-1910d-zukuenftigen-chancen-psychoanalytischentherapie

Geller, J. D., Norcross, J. C., \& Orlinsky, D. E. (2005). The Psychotherapist's Own Psychotherapy: Patient and Clinician Perspectives. Oxford University Press.

Hahn, D., Weck, F., Witthöft, M., \& Kühne, F. (in prep.). What Characterizes Helpful Personal Practice in Psychotherapy Training? Results of an Online Survey.

Hippler, B., \& Görlitz, G. (2001). Selbsterfahrung in der Gruppe: Person- und patientenorientierte Übungen. Klett-Cotta.

Ieva, K. P., Ohrt, J. H., Swank, J. M., \& Young, T. (2009). The impact of experiential groups on master students' counselor and personal development: a qualitative investigation. The Journal for Specialists in Group Work, 34, 351-368. https://doi. org/10.1080/01933920903219078

Kanfer, F. H., Reinecker, H., \& Schmelzer, D. (2012). Selbstmanagement-Therapie: Ein Lehrbuch für die klinische Praxis (5., korr. und durchges. Aufl). Springer Medizin.

Kivlighan, M. D., Adams, M. C., Obrecht, A., Kim, J. Y. C., Ward, B., \& Latino, C. A. (2019). Group therapy trainees' social learning and interpersonal awareness: the role of cohesion in training groups. Journal for Specialists in Group Work, 44, 62-76. https://doi.org/10.1080/01933922.2018.1561777

Kline, W. B., Falbaum, D. F., Pope, V. T., Hargraves, G. A., \& Hundley, S. F. (1997). The significance of the group experience for students in counselor education: a preliminary naturalistic inquiry. Journal for Specialists in Group Work, 22, 157-166. https://doi.org/10.1080/01933929708414377

Krogel, J. A. (2009). The Group Questionnaire: A New Measure of the Group Relationship. Brigham Young University.

Laireiter, A.-R., \& Willutzki, U. (2003). Self-reflection and self-practice in training of cognitive behaviour therapy: an overview. Clinical Psychology \& Psychotherapy, 10, 19-30. https://doi.org/10.1002/cpp.348

Leitemo, K., Vestbø, H. S. B., Bakali, J. V., \& Nissen-Lie, H. A. (2020). The role of attachment anxiety and avoidance for reduced interpersonal problems in training group analytic therapy. Group Dynamics: Theory, Research, and Practice, 24, 26-41. https://doi.org/10.1037/gdn0000112

Lo Coco, G., Tasca, G. A., Hewitt, P. L., Mikail, S. F., \& Kivlighan, Jr, D. M. (2019). Ruptures and repairs of group therapy alliance. An untold story in psychotherapy research. Research in Psychotherapy: Psychopathology, Process and Outcome, 22. https://doi.org/10.4081/ripppo.2019.352

Luke, M., \& Kiweewa, J. M. (2010). Personal growth and awareness of counseling trainees in an experiential group. Journal for Specialists in Group Work, 35, 365-388. https://doi.org/10.1080/01933922.2010.514976

Malikiosi-Loizos, M. (2013). Personal therapy for future therapists: reflections on a still debated issue. European Journal of Counselling Psychology, 2, 33-50. https://doi.org/10.5964/ejcop.v2i1.4

McCarthy, C. J., Falco, L. D., \& Villalba, J. (2014). Ethical and professional issues in experiential growth groups: moving forward. Journal for Specialists in Group Work, 39, 186-193. https://doi.org/10.1080/01933922.2014.924722

Moller, N. P., \& Rance, N. (2013). The good, the bad and the uncertainty: trainees' perceptions of the personal development group. Counselling and Psychotherapy Research, 13, 282-289. https://doi.org/10.1080/14733145.2012.754482 
Murphy, B. A., Costello, T. H., Watts, A. L., Cheong, Y. F., Berg, J. M., \& Lilienfeld, S. O. (2018). Strengths and weaknesses of two empathy measures: a comparison of the measurement precision, construct validity, and incremental validity of two multidimensional indices. Assessment, 107319111877763. https://doi.org/10.1177/1073191118777636

Ogrodniczuk, J. S., \& Piper, W. E. (2003). The effect of group climate on outcome in two forms of short-term group therapy. Group Dynamics: Theory, Research, and Practice, 7, 64-76. https://doi.org/10.1037/1089-2699.7.1.64

Oh, S., Mitchell, M. D., McKinzie Bennett, C., Rendon Finnell, L., Saliba, Y., Heard, N. J., \& Pennock, E. R. (2018). Journal sharing on group cohesion and goal attainment in experiential growth groups. Journal for Specialists in Group Work, 43, 206-229. https://doi.org/10.1080/01933922.2018.1484541

Ohrt, J. H., Prochenko, Y., Stulmaker, H., Huffman, D., Fernando, D., \& Swan, K. (2014). An exploration of group and member development in experiential groups. Journal for Specialists in Group Work, 39, 212-235. https://doi.org/10.1080/ 01933922.2014.919047

Orlinsky, D. E., Botermans, J. F., \& Rønnestad, M. H. (2001). Towards an empirically grounded model of psychotherapy training: four thousand therapists rate influences on their development. Australian Psychologist, 36, 139-148. https://doi. org/10.1080/00050060108259646

Orlinsky, D. E., Norcross, J., Rønnestad, M. H., \& Wiseman, H. (2005). Outcomes and impacts of the psychotherapists' own psychotherapy: a research review. In J. D. Geller, J. Norcross, \& D. Orlinsky (eds), The Psychotherapist's Own Psychotherapy: Patient and Clinician Perspectives (1st edn, pp. 214-230). Oxford University Press.

RStudio Team (2015). RStudio: Integrated Development for R. RStudio, Inc. http://www.rstudio.com/

Safran, J. D., Muran, J. C., \& Eubanks-Carter, C. (2011). Repairing alliance ruptures. Psychotherapy, 48, 80-87. https://doi. org/10.1037/a0022140

Sandell, R., Carlsson, J., Schubert, J., Grant, J., Lazar, A., \& Broberg, J. (2006). Therapists' therapies: the relation between training therapy and patient change in long-term psychotherapy and psychoanalysis. Psychotherapy Research, 16, 306-316. https://doi.org/10.1080/10503300500273110

Schneider, K., \& Rees, C. (2012). Evaluation of a combined cognitive behavioural therapy and interpersonal process group in the psychotherapy training of clinical psychologists: evaluation of psychotherapy training. Australian Psychologist, 47, 137146. https://doi.org/10.1111/j.1742-9544.2012.00065.x

Strauß, B., \& Mattke, D. (eds) (2018). Gruppenpsychotherapie: Lehrbuch für die Praxis. Springer Berlin Heidelberg. https:// doi.org/10.1007/978-3-662-54644-4

Sunderji, N., Malat, J., \& Leszcz, M. (2013). Group day: experiential learning about group psychotherapy for psychiatry residents at University of Toronto. Academic Psychiatry, 37, 352. https://doi.org/10.1176/appi.ap.12050096

Tschuschke, V., \& Greene, L. R. (2002). Group therapists' training: what predicts learning? International Journal of Group Psychotherapy, 52, 463-482. https://doi.org/10.1521/ijgp.52.4.463.45522

Tschuschke, V., Meier, U., \& Theilacker, M. (2011). Gruppenselbsterfahrung in der psychotherapeutischen Ausbildung. Psychotherapeutenjournal, 3, 7.

Yalom, I. D., \& Leszcz, M. (2008). The Theory and Practice of Group Psychotherapy. Hachette UK.

Young, T. L., Reysen, R., Eskridge, T., \& Ohrt, J. H. (2013). Personal growth groups: measuring outcome and evaluating impact. Journal for Specialists in Group Work, 38, 52-67. https://doi.org/10.1080/01933922.2012.745915

Zhu, P. (2018). Experiential growth group in counselor education: a review of its pedagogy, research, and ethical dilemmas. Journal for Specialists in Group Work, 43, 144-165. https://doi.org/10.1080/01933922.2018.1451581

Cite this article: Maaß U, Kühne F, Hahn D, and Weck F (2022). Group cohesion in group-based personal practice. Behavioural and Cognitive Psychotherapy 50, 28-39. https://doi.org/10.1017/S1352465821000369 